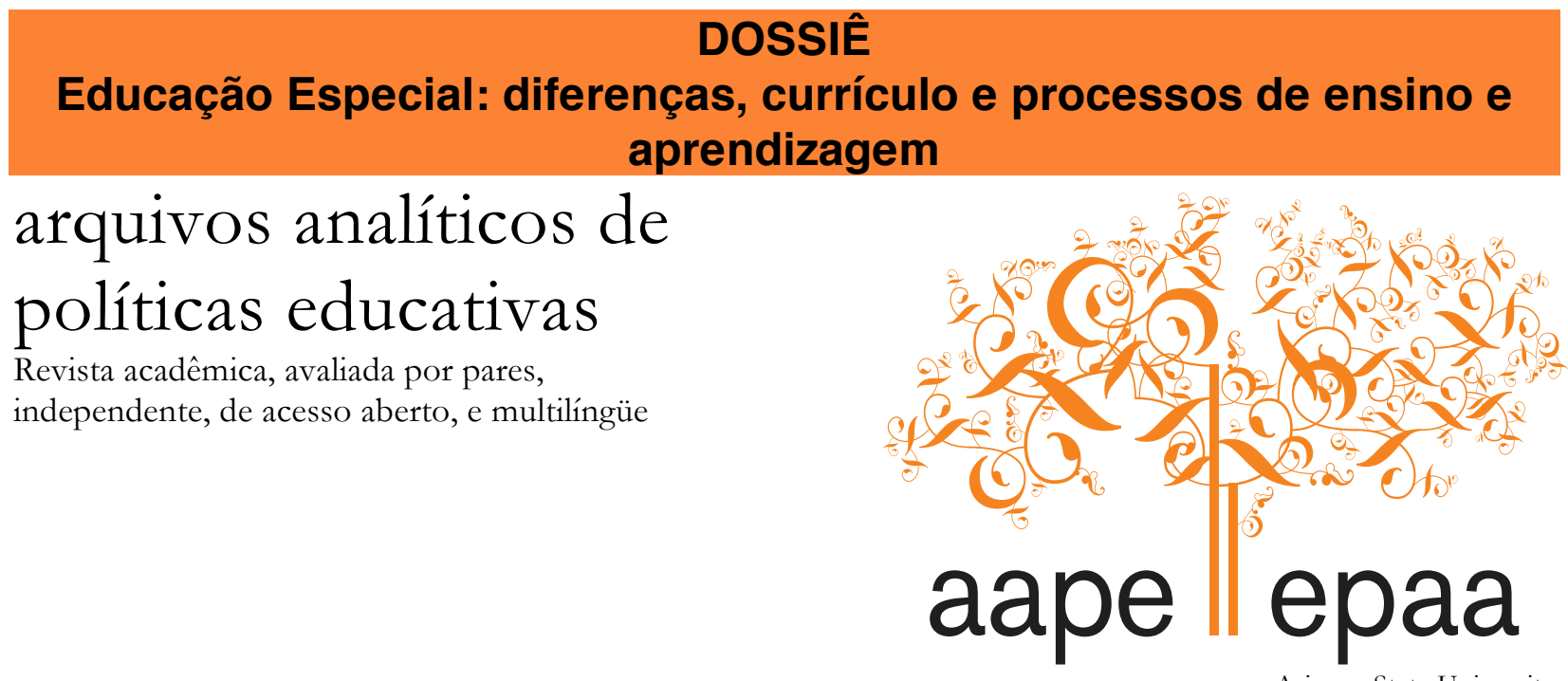

Arizona State University

Volume 22 Número $84 \quad 11$ de agosto de 2014

ISSN 1068-2341

\title{
Autismo: a educação infantil como cenário de intervenção
}

\author{
Débora R. P. Nunes \\ \&o \\ Eliana Rodrigues Araújo \\ Universidade Federal do Rio Grande do Norte-UFRN \\ Brasil
}

Citação: Nunes, D. R.P.; Araújo, E.R. (2014). Autismo: a educação infantil como cenário de intervenção. Arquivos Analíticos de Políticas Educativas, 22(84). Dossiê Educação Especial: diferenças, curriculo e processos de ensino e aprendizagem. Editoras convidadas: Márcia Denise Pletsch \& Geovana Mendonça Lunardi Mendes. http://dx.doi.org/10.14507/epaa.v22n84.2014

Resumo: Nas últimas décadas, a eficácia dos programas de intervenção, em autistas, na primeira infância, se constitui objeto de pesquisa amplamente investigado. Os pressupostos teóricos da neuroplasticidade e abordagens desenvolvimentistas são factuais quanto à importância do tratamento precoce e intensivo para essa população. Nessa perspectiva, estudos indicam a necessidade de serem incluídas crianças, antes dos cinco anos de idade, em programas de intervenção com carga-horária de, no mínimo, 25 horas semanais. No caso, o ensino infantil é um segmento educacional altamente promissor, na implementação de práticas interventivas focadas nesse contingente infantil. Além de atender alunos de 0 a 5 anos, em regime de horário integral ou parcial, o ensino infantil tem como propósito promover o desenvolvimento integral do educando, contemplando aspectos físicos, psicológicos, intelectuais e sociais. O presente artigo tem como objetivo descrever os fundamentos da intervenção precoce, com ênfase nas melhores práticas interventivas, direcionadas para populações com autismo. Como complemento, discute-se a relevância dessa modalidade de atendimento no contexto nacional da educação infantil.

Palavras-chave: autismo; educação infantil; intervenção precoce.

Página web: http://epaa.asu.edu/ojs/

Facebook: /EPAAA

Artigo recebido: $28 / 02 / 2014$

Twitter:@epaa_aape

Revisões recebidas: 10/06/2014

Aceito: $28 / 06 / 2014$ 


\section{Autism: early childhood education as an intervention scenario}

Abstract: In recent decades, the effectiveness of early intervention programs for children with autism has been widely investigated. The importance of early and intensive care for this population is grounded in principles of neuroplasticity and developmental theories. The scientific literature documents the need for including children younger than five in early intervention programs, for a minimum of 25 hours per week. In this case, early childhood education settings and preschools become highly-promising educational scenarios for implementing intervention. In addition to working with children (birth-5) in full or part-time schedules, these educational programs focus on the integral development of the child, including physical, psychological, intellectual, and social aspects. The purpose of the current article is to describe the principles of early intervention, with an emphasis on best practices directed toward people with autism. Additionally, the relevance of this type of intervention in the context of early childhood education settings in Brazil is further discussed.

Keywords: autism; early intervention; early childhood education.

\section{Autismo: la educación de la primera infancia como un escenario de intervención}

Resumen: En las últimas décadas, la eficacia de los programas de intervención para autistas en la primera infancia, ha sido un objeto ampliamente investigado. Las bases teóricas de la neuroplasticidad y del desarrollo enfatizan la importancia de intervenir precozmente con esta populación. En esta perspectiva, estudios indican la necesidad de incluir niños antes de los cinco años de edad, en programas de intervención con carga horaria mínima de 25 horas por semana. La Educación preescolar es un segmento educacional altamente promisor en la implementación de prácticas interventoras para esta populación. Además de atender alumnos de 0 a 5 años de edad, en periodo integral o parcial, la educación infantil asume como meta promover o desarrollo integral del educando, contemplando aspectos físicos, psicológicos, cognitivos y sociales. El presente artículo tiene como objetivo describir los fundamentos de la intervención precoz, con foco en las mejores prácticas interventoras, direccionadas para populaciones con autismo. Como complemento, se discutirá la relevancia de esta modalidad de atendimiento en el contexto Nacional de la Educación infantil.

Palabras-clave: autismo; educación infantil; intervención temprana

\section{Introdução}

As últimas décadas foram marcadas por intensas discussões acerca do autismo, tanto no meio acadêmico como nas mídias sociais. Pesquisas científicas abrangendo desde fatores etiológicos até a escolarização da população afetada são conduzidas nas mais diversas áreas do conhecimento (Schmidt, 2013; Schwartzman \& Araújo, 2011). Destacam-se, ainda, a produção de livros, filmes e, até telenovelas, abordando essa temática. Esse fenômeno impulsionou a conscientização da sociedade sobre as especificidades dessa síndrome, notadamente em relação aos aspectos de identificação e intervenção precoce (Johnson \& Myers, 2007; Matson \& Konst, 2013).

$\mathrm{Na}$ mais recente classificação do Manual Diagnóstico e Estatístico de Transtornos Mentais, o DSM-V (APA, 2013), o autismo é descrito como um distúrbio do neurodesenvolvimento e denominado de Transtorno do Espectro do Autismo (TEA). Prejuízos sociocomunicativos e a manifestação de padrões restritos de comportamentos e interesses são as principais características dessa condição, cuja incidência é maior em homens do que em mulheres (APA, 2013). A prevalência para TEA é estimada em um a cada 88 crianças (Centers for Disease Control and Prevention, 2012 apud Brasil, 2013), o que sustenta a afirmativa de que o autismo se tornou um dos 
transtornos do desenvolvimento mais comuns na atualidade (Fombonne, 2009). Na América do Sul, o único estudo publicado até o presente momento foi realizado em Atibaia (SP), indicando a taxa de incidência de uma pessoa em cada grupo de 330 (Paula et al., 2011).

Até presentemente, não há perspectiva de cura para o autismo. Os sintomas podem, no entanto, ser minimizados por meio de programas de intervenção psicoeducacionais (Matson \& Konst, 2013; National Research Council, NRC, 2001;). Resultados de estudos sugerem que as práticas interventivas, conduzidas com pré-escolares (menores de 5 anos de idade) tendem a gerar dados mais promissores que os programas envolvendo indivíduos mais velhos (Matson \& Konst, 2013; NRC, 2001). Adicionalmente, algumas pesquisas salientam que, independentemente da abordagem teórica, os tratamentos devem ser de caráter intensivo, com duração média de 25-40 horas semanais (Matson \& Konst, 2013; NRC, 2001).

Nesse cenário a educação infantil pode configurar-se como um espaço propício às ações de intervenção psicoeducacional. Além de atender crianças de 0 a 5 anos de idade, o período de permanência desses indivíduos na escola é intensivo, podendo variar de 20 a 35 horas semanais.

\section{Intervenção precoce: um breve histórico}

A intervenção precoce é conceituada como um sistema coordenado de serviços com o objetivo de promover o desenvolvimento da criança de zero a cinco anos de idade. Dentre as metas desse sistema configuram-se a estimulação das competências da criança, a minimização ou remediação de suas incapacidades e o empoderamento da família (Byington \& Whitby, 2011). O modelo da intervenção precoce está ancorado na ideia de que as crianças com deficiência e outras necessidades especiais diferem, de algum modo, daquelas com desenvolvimento típico (Mendes, 2010; Nunes, 1995). Essas diferenças individuais desencadeiam demandas de oferta de modalidades de serviços de atendimentos específicos e interdisciplinares.

Os programas de Intervenção Precoce na Infância (IPI) - inicialmente denominados de estimulação precoce- surgiram na década de 1960, a partir de uma evolução natural dos programas de educação especial. De acordo com Love \& colaboradores (2008), em 1965, o governo americano deu início ao movimento de estimulação precoce com o programa Head Start, cujo objetivo era potencializar as competências cognitivas, intelectuais e sociais, bem como a saúde física e mental de crianças oriundas de famílias com fatores de risco econômico, social, de saúde e/ou de saúde mental. Esse programa, de abordagem médico-terapêutica, tinha caráter claramente compensatório e oferecia apoio em múltiplas áreas, incluindo aspectos sociais, educacionais e da saúde para crianças da educação infantil e ensino fundamental, previamente inscritas no Head Start ou em outros programas educacionais. Apesar do sucesso que tiveram, mais especificamente nos aspectos comportamentais, os programas atuavam isoladamente no problema da criança, não considerando a família e o ambiente que a envolvia (Sandall et al., 2000, 2005, grifo nosso).

No Brasil, os programas de IPI alcançaram notoriedade nas décadas de 1970 e 1980. Seguindo abordagem médica, esses programas se vinculavam a hospitais, serviços de saúde e instituições especializadas (Cunha \& Benevides, 2012). Pautados no modelo norte-americano, os programas de IPI eram de caráter compensatório e, essencialmente, centrados nos déficits da criança, conforme já assinalado. O objetivo consistia em prevenir deficiências secundárias e reabilitar o indivíduo em processo de desenvolvimento.

A partir da década de 1990, a literatura internacional registrou uma redefinição no foco de atuação dos programas de IPI (Bolsanello, 2003; Byington \& Whitby, 2011). Observou-se, nesse novo cenário, a participação mais expressiva das famílias, priorizando o desenvolvimento de suas competências para lidar com a criança com deficiência. Nessa abordagem, por deslocar a unidade de 
intervenção da criança para a família, o termo "estimulação precoce" foi substituído por "intervenção precoce" (Almeida, 2004). Os programas de IPI passaram a se configurar como um conjunto de intervenções dirigidas à população infantil, à família e ao contexto, tendo por objetivo "responder às necessidades transitórias ou permanentes que afetam crianças de risco ou aquelas com transtornos de desenvolvimento" (Gat, 2000; apud Soejima \& Bolsanello, 2012, p.66). Essas intervenções teriam como propósito promover e potencializar o desenvolvimento da criança, viabilizando a sua integração no ambiente familiar, escolar e social (Soejima \& Bolsanello, 2012). Em última instância, como salienta Almeida (2004), o propósito seria tornar a família mais autônoma, capaz de gerir os próprios recursos de que precisa, enquanto agente receptor e ativo do programa de IPI.

Inspirado em uma perspectiva ecossistêmica, difundida por Bronfenbrenner (2005), os programas de IPI passaram a privilegiar o contexto natural como foco de intervenção, as potencialidades da criança e o emprego de práticas adequadas ao seu nível de desenvolvimento. Essa perspectiva ancora-se na ideia de que o maior impacto no curso da vida de uma criança seria a sua participação, no decorrer das atividades cotidianas, nas interações com as pessoas em torno dela mesma (Bronfenbrenner, 2005). Essa perspectiva contempla as constelações da família, dos pares, da escola e da comunidade como esferas de influência sobre a criança (Gable, 2006). Esse posicionamento compreende, ainda, o desenvolvimento como um processo que envolve estabilizações e mudanças das características biopsicológicas de um ser humano, não apenas ao longo da vida, mas também, através de gerações (Bronfenbrenner, 2005).

Assim, o novo modelo de IPI preconizou o uso de um currículo desenvolvimental e funcional, aplicado nas oportunidades naturais de aprendizagem do indivíduo. Dessa forma, Briker (2001) e Dunst \& Bruder (2002) indicam que os contextos de intervenção passaram a ser a casa, a comunidade, a creche e quaisquer outros locais, cenários e atividades nos quais participam crianças da mesma idade, livres de deficiências.

Seguindo esse norteamento, os Estados Unidos e a Europa redefiniram as diretrizes voltadas às práticas educacionais de crianças de zero a cinco anos de idade (Soejima \& Bolsanello, 2012). Nos Estados Unidos, por exemplo, a seção de Intervenção Precoce do Council for Exceptional Children ${ }^{1}$ publicou, em 2000, um conjunto de orientações para famílias e profissionais que atuam com crianças com deficiência (Sandall, Mclean \& Smith, 2000). Tendo como fundamento os resultados de pesquisas científicas, esse documento sinaliza a importância de modelos interdisciplinares de avaliação e de intervenção focados nas demandas da criança e de sua família. A avaliação interdisciplinar é essencial para detectar o foco e o contexto de intervenção. As práticas focadas nas demandas da criança dizem respeito a como, onde e quando a intervenção ocorrerá. Adicionalmente, essas mesmas práticas determinam a forma como o desempenho da criança será monitorado. Trata-se de um elemento fundamental para definir se as práticas interventivas adotadas serão mantidas ou modificadas, assim como se novos focos de intervenção são detectados.

O foco na família refere-se ao provimento de recursos e suportes necessários para que os cuidadores disponham de tempo, energia, conhecimento e habilidades para viabilizar as oportunidades de aprendizagem essenciais para o desenvolvimento da criança (Sandall, Mclean \& Smith, 2000). É interessante ressaltar que esse movimento de apoio familiar deu origem ao termo empoderamento (Williams \& Aiello, 2001), que alude ao conceito de capacitação/fortalecimento da família, como parte interessada ativa no processo de intervenção. A família é empoderada quando o sucesso de suas conquistas é atribuído, majoritariamente, ao seu próprio desempenho.

\footnotetext{
1 Trata-se de uma organização nacional, fundada em 1973, dedicada a promover e difundir práticas cientificamente válidas, assim como políticas de apoio a crianças de $0-8$ anos com deficiências, atrasos ou risco de desenvolvimento e suas famílias (Sandall, Mclean \& Smith, 2000).
} 
Assim como nos Estados Unidos, uma mudança de foco nos programas de IPI foi, também, observada em diversos países da Europa. De forma específica, os programas de IPI de 26 países europeus, analisados no período 2005-2010, indicaram a criança, sua família e o meio em que vive como alvo de intervenção. Essa mudança corresponde a uma evolução das ideias de IPI no campo da deficiência (incapacidade), especialmente quanto ao paradigma do modelo "médico" para um modelo "social” (European Agency for Development in Special Needs Education, 2010).

Apesar das pesquisas internacionais sinalizarem a importância das práticas interventivas precoces centradas na família, no desenvolvimento das potencialidades da criança e no contexto, são poucos os estudos brasileiros que seguem essas diretrizes (Bonsanello, 2003). Como constatam Williams \& Aiello (2001), o limitado número de investigações sobre os programas de IPI são tipicamente encontrados em teses e dissertações não publicadas. A participação dos pais se limita em fornecer informações ou levantar suas expectativas sobre a temática. A participação de estimuladores precoces em ambientes escolares é escasso no Brasil (Pereira \& Grave, 2012). Por fim, é limitado o número de estudos conduzidos em contextos naturais de ensino, envolvendo professores como agentes de intervenção (Dall' Aqua, Takiuchi \& Zorzi, 2008; Nunes, et. al., 2013).

\section{Melhores práticas em programas de IPI para crianças com autismo}

Em termos de abordagens teóricas, os programas de IPI para populações com autismo, descritos na literatura, podem ser inseridos em um continuum, no qual, em uma extremidade se situa o paradigma desenvolvimentista, e na outra, o paradigma comportamental. A seguir, seguem-se breves explanações acerca desses dois modelos paradigmáticos.

A abordagem desenvolvimentista tem como característica central compreender as singularidades e desvios do desenvolvimento da criança com autismo, a partir ou à luz do desenvolvimento típico, sem uma visão determinista (Lampreia, 2007). De acordo com Greenspan $\& W i e d e r(1997)$, essa abordagem baseia-se na suposição de que a sintomatologia autista seja provocada pelas dificuldades de processamento, de base biológica. Assim, prejuízos na modulação sensorial ou na coordenação motora da criança poderiam impactar sua aprendizagem e a qualidade de suas interações socioafetivas.

Os programas de tratamento baseados na abordagem desenvolvimentista têm como objetivo ajudar a criança a contornar as suas próprias dificuldades de processamento para restabelecer o contato afetivo. No caso, as habilidades sociocomunicativas são, especificamente, os focos de intervenção. Defensores dessa abordagem supõem que o desenvolvimento individual e a aprendizagem sejam processos complexos e dinâmicos que ocorrem dentro de um contexto social (Lampreia, 2007). Nessa perspectiva, as ações interventivas acontecem, tipicamente, em contextos naturais, envolvendo familiares, professores ou outros indivíduos próximos da criança.

Dentre os programas educacionais com base no paradigma desenvolvimentista, destacam-se o Denver Model e o Developmental, Individual-difference, Relationship-based Model (DIR) (modelo desenvolvimentista, de diferença individual, baseado no relacionamento) (Lampreia, 2007; NRC, 2001; Simpson, 2005). O primeiro, originado em 1981 na Universidade do Colorado (EUA), traz como premissa que o jogo é um veículo fundamental para a aprendizagem de habilidades sociais, emocionais, comunicativas e cognitivas. Como objetivos gerais, o programa visa aumentar os níveis cognitivos, particularmente na área das funções simbólicas; ampliar a comunicação por meio de gestos, sinais e palavras; e potencializar o crescimento social e emocional através de relações interpessoais com adultos e pares (NRC, 2001). Nas últimas décadas, diversos estudos têm sido publicados evidenciando os efeitos promissores desse modelo em crianças com autismo, de 12 a 48 
meses de idade (Dawson et al., 2010; NRC, 2001; Rogers et al., 2012; Vismara, Colombi, Rogers, 2009).

O segundo modelo (DIR), elaborado por Stanley Greenspan, na década de 1990, preconiza que a aprendizagem da criança é viabilizada por fatores intrínsecos, como a motivação, o desejo de explorar o ambiente e pelas interações socioafetivas, realizadas em contextos naturais com os cuidadores (Thompson, 2011). Por meio de interações lúdicas, dirigidas pela criança, esse modelo de intervenção visa auxiliar o indivíduo a reestabelecer a sequencia de desenvolvimento que fora interrompida (Simpson, 2005; Lampreia, 2007). Um dos elementos essenciais desse programa é o Floortime, compreendido tanto como um tratamento, como uma filosofia. Enquanto prática interventiva, ofloortime, que pode ser aplicado no ambiente familiar, escolar ou clínico, alude à ideia de que as brincadeiras e atividades de aprendizagem devem ocorrer "no chão". Embora as pesquisas sobre a eficácia desse modelo sejam, ainda, incipientes, os poucos estudos conduzidos na área evidenciam resultados promissores (Mahony; Perales, 2003; Pajareya, K; Nopmaneejumruslers, 2011; Simpson, 2005)

Os modelos de intervenção ancorados no paradigma comportamental trazem como base os pressupostos teóricos de Watson e Skinner (Simpson, 2005). Nessa abordagem, os comportamentos desadaptativos não são interpretados como sintomas de uma doença, mas como eventos passíveis de modificação. Assim, o processo de intervenção consiste em identificar as relações funcionais que ocorrem entre os eventos ambientais antecedentes e as respostas do organismo ou eventos consequentes. Para identificar essas relações, deve-se especificar a ocasião em que a resposta ocorre, a própria resposta, e as consequências reforçadoras que a mantém (Prizant \& Meyer, 2003). Em contraste com a abordagem desenvolvimentista, as intervenções comportamentais, tipicamente ocorrem em ambientes mais estruturados.

Dentre as práticas interventivas baseadas no modelo comportamental destaca-se o Learning Experience: An Alternative Program for Preschoolers and Parents ${ }^{2}$ (LEAP), criado em 1982, na Universidade de Colorado. O LEAP é um programa educacional desenvolvido na pré-escolar, assim como um modelo interventivo destinado aos pais. Ele foi um dos primeiros programas norte-americanos a defender a inserção de crianças com autismo em contextos regulares de ensino. No cenário da escola comum, a mediação feita pelos pares com desenvolvimento típico é um dos pontos críticos do programa. O currículo escolar desenvolvido no LEAP é individualizado e traz como metas o desenvolvimento de habilidades sociocomunicativas e competências comportamentais que favorecem a independência da criança em idade pré-escolar (Simpson, 2005). O envolvimento familiar é imprescindível. Assim, em paralelo ao programa escolar, os pais são capacitados a empregarem estratégias de ensino e de manejo de comportamento com os filhos no contexto domiciliar.

A literatura científica tem produzido um número expressivo de estudos revelando a eficácia do LEAP em crianças com autismo (ver Simpson, 2005 para revisão). Dentre os ganhos evidenciados estão a redução de comportamentos inadequados, a melhoria na interação com pares e o maior desenvolvimento cognitivo e da linguagem (Strain; Hoyson, 2000; Simpson, 2005; Strain; Bovey, 2011).

Os resultados conclusivos dos estudos sobre IPI sugerem não haver uma única abordagem que possa ser aplicada a todas as crianças com TEA (Thompson, 2011), mas identificam alguns elementos universais considerados como melhores práticas no tratamento precoce desse contingente infantil. Assinale-se que o conceito de melhores práticas origina-se da área de Administração e refere-se às estratégias de produção que proveem melhoria de desempenho. Melhores práticas também podem ser compreendidas como a utilização de processos reconhecidos como o melhor método de

\footnotetext{
${ }^{2}$ Experiência de aprendizagem: um programa alternativo para pais e crianças pré-escolares.
} 
conduzir uma ação, levando a organização, comprovadamente, a resultados superiores (Davies \& Kochar, 2002). Em educação especial, as melhores práticas se constituem em modalidades de intervenções mais eficazes e eficientes e que asseguram um desempenho ótimo do aluno (Spaulding, 2009).

As melhores práticas em IPI para crianças com autismo preconizam que o tratamento cumpra os seguintes requisitos: a) seja destinado aos indivíduos de zero a cinco anos de idade, que estejam em risco de atraso ou desvio em seu desenvolvimento (Lampreia, 2007; NRC, 2001); b) seja baseado no perfil individual da criança (NRC, 2001; Thompson, 2011); c) tenha enfoque no desenvolvimento de cinco domínios, a saber: atenção a elementos do ambiente, imitação, compreensão e uso da linguagem, jogo apropriado com brinquedos e interação social (Dawson \& Osterling, 1998; NRC 2001); d) analise os fatores de estresse familiar, conciliando os apoios necessários - sociais, clínicos e terapêuticos (Guralnick, 1998); e) envolva os pais como parceiros e agentes ativos na intervenção, que deve ocorrer no início de vida da criança, intensivamente (20-40h semanais) (Girolametto et al., 2006; Greenspan \& Wieder, 1999; Guralnick,1998; Sussman, 1999; McConachie et al., 2005; NRC, 2001).

\section{A Educação Infantil como contexto de programas de IPI}

Ao longo da história, o atendimento a menores de cinco anos de idade que evidenciavam necessidades educacionais especiais, eram ofertados separadamente daqueles serviços destinados às crianças com desenvolvimento típico (Mendes, 2010). Na atualidade, ancorado no paradigma da inclusão, é preconizado que os programas de IPI sejam operacionalizados nos espaços inclusivos da Educação Infantil, como as creches e pré-escolas. Como argumenta Nunes (1995), a creche é, por excelência, um dos espaços para o desenvolvimento de ações preventivas primárias. Dentre essas ações configuram-se os programas educacionais para crianças com riscos para a excepcionalidade.

As primeiras creches brasileiras foram criadas, no final do século XIX, com o propósito de atender filhos de mães solteiras, abandonadas ou viúvas, que não tinham condições de cuidar de suas próprias crianças (Mariotto, 2003; Oliveira, 1988; Pacheco \& Dupret, 2004). Em uma perspectiva médica e sanitarista, essas instituições, de cunho primordialmente filantrópico, objetivavam "nutrir as crianças, promover a saúde e difundir normas rígidas de higiene, associando à pobreza a falta de conhecimentos de puericultura e abafando qualquer relação com as questões econômicas e políticas do país" (Andrade, p. 137).

Nas décadas de 1920 e 1930, com o crescimento da industrialização no país e a expressiva inserção da mulher no mercado de trabalho, observa-se a participação mais ativa do Estado na criação e manutenção dessas instituições. As duas décadas posteriores foram marcadas pelo desenvolvimento de programas governamentais que priorizavam a alimentação e higiene da mulher trabalhadora e de seus filhos (Andrade, 2010; Mariotto, 2003; Oliveira, 1988; Pacheco \& Dupret, 2004).

O modelo de atendimento higienista é substituído pelo paradigma da "compensação", na década de 1960. Influenciado pelos pressupostos teóricos de privação materna de Bowlby ${ }^{3}$, a creche torna-se um espaço que visa o desenvolvimento infantil, compensando o precário meio em que as famílias das classes trabalhadoras viviam (Andrade, 2010; Mariotto, 2003; Oliveira, 1988; Pacheco \& Dupret, 2004). Nessa perspectiva, a creche, que atendia essencialmente populações de baixa renda,

\footnotetext{
${ }^{3}$ Nesse período era prevalente a ideia de que a ausência da relação afetiva mãe-criança culminaria em prejuízos físicos, mentais e sociais da criança (Mendes, 2010).
} 
passa a configurar-se como um local para compensar deficiências biopsicoculturais, em relação ao padrão da criança de classe média (Mendes, 2010).

Foi apenas no final da década de 1980 e nos anos 90, que o atendimento à criança de zero a seis anos ${ }^{4}$ passou a ser vinculado ao sistema de ensino e garantido por lei. Assim, a Constituição de 1988 estabelece que esse atendimento deva contemplar não apenas a tarefa de cuidar, mas também de educar. Nessa perspectiva, a Lei de Diretrizes e Bases da Educação Nacional (LDB/9.394) define as creches e pré-escolas como a primeira etapa da educação básica, que tem como finalidade complementar a ação da família e da comunidade. Embora essa etapa de escolarização não seja obrigatória, ela traz como propósito o desenvolvimento integral da criança, incluindo seus aspectos físicos, psicológicos, intelectuais e sociais (Alves; Veríssimo, 2007; Andrade, 2010).

De acordo com o Referencial Curricular Nacional para a Educação Infantil, o que se pretende na educação infantil é garantir as melhores oportunidades de desenvolvimento a todas as crianças, incluindo aquelas que evidenciam necessidades educacionais especiais (Brasil, 2000). Adicionalmente, conforme reza a Política Nacional de Educação Infantil, a educação de crianças que apresentam excepcionalidades deve ser realizada em conjunto com seus pares com desenvolvimento típico (Brasil, 2006). Nessa perspectiva, torna-se relevante desenvolver programas interventivos, como o IPI, para essa população em contextos comuns da educação infantil.

Dada a recência do paradigma da Educação Inclusiva e da nova concepção da creche, como entidade educacional, é compreensível haver resistências a essa proposta. Em estudo conduzido por Nunes (1996), por exemplo, funcionários de creches fluminenses declararam não ter condições de receber crianças com deficiência por não disporem de recreadores e profissionais capacitados. Adicionalmente, alguns verbalizaram que as crianças deveriam ser atendidas em clínicas e que a creche não era um "hospital".

É importante argumentar, no entanto, que os objetivos dos programas de IPI e da educação infantil são convergentes. Isto é, ambos fomentam o desenvolvimento global da criança (Soejina \& Bonsanello, 2012). O mesmo Referencial Curricular Nacional (Brasil, 1998) para a educação, recomendado para alunos com desenvolvimento típico é fundamental para aqueles que apresentam alterações significativas no processo de desenvolvimento e aprendizagem (Pereira \& Grave, 2012). Nesse aspecto, os educadores ou professores de creches e pré-escolas devem estar capacitados em atender as demandas dos alunos que evidenciam necessidades educacionais especiais, incluindo-se aqueles com sintomas do autismo, mesmo sem diagnóstico efetivado.

Nunes (1995) salienta a importância em criarem-se programas específicos de IPI em creches. Para que essa proposta seja operacionalizada são necessárias mudanças políticas e educacionais significativas na educação infantil. Primeiramente, observa-se que a presença da criança com deficiência na Educação Infantil é inexpressiva, representando apenas 1\% das matrículas efetuadas nessa etapa de ensino (Bueno \& Meletti, 2011). Adicionalmente, embora preconizado por lei, a oferta de serviços de educação especial é, ainda, escassa nas creches e pré-escolas (Pereira \& Matsukura, 2013). A presença de agentes de intervenção precoce na escola é limitada (Nunes et al., 2013; Pereira \& Grave, 2012;). Carecemos, ainda, do conhecimento necessário para saber o modo como deve ser realizada a formação de professores da Educação Infantil que atuam com alunos com necessidades educacionais especiais. Para crianças com autismo, salienta-se, ainda, o fato de não haver no país serviços públicos em atendimentos de IPI para essa população, apesar do Ministério da Saúde reconhecer que "a intervenção precoce em casos de TEA têm maior eficácia e contemplam maior economia" (Brasil, 2013 p.20). Por fim, o Brasil carece de estudos científicos que avaliem o

\footnotetext{
${ }^{4} \mathrm{Na}$ atualidade, o Estado determina que o atendimento 0-3 anos seja ofertado em creches e de 4-5 anos na pré-escola. Aos seis anos, a criança ingressaria no Ensino Fundamental.
} 
processo de inclusão de crianças com deficiência na educação infantil (Mendes, 2010; Pereira \& Matsukura, 2013), particularmente aquelas com diagnóstico de autismo (Nunes et al., 2013).

\section{Considerações finais}

O presente artigo abordou os fundamentos da Intervenção Precoce na Infância (IPI), tendo como destaque as melhores práticas interventivas destinadas a educandos com autismo. Considerando-se a atual política educacional inspirada no paradigma da inclusão, foram apresentados argumentos sobre a operacionalização dos programas de IPI nas classes comuns da Educação Infantil, particularmente, no contexto da creche.

Mudanças expressivas nas concepções de programas de IPI foram observadas nas últimas décadas. Nesse aspecto, o enfoque de modelo médico ou patológico, pautado nos prejuízos da criança, foi gradativamente e, aparentemente, substituído por uma abordagem interventiva de cunho social, contemplando assim a família e o meio ambiente em sentido amplo. No caso, o empoderamento dos cuidadores, a ênfase nas potencialidades do indivíduo com deficiência e a utilização de contextos naturais, como a casa e a escola, como cenários de intervenção são características basilares desse novo modelo paradigmático. Apesar dos resultados das pesquisas científicas internacionais revelarem os dados promissores sobre essa prática, no Brasil, os modelos de IPI continuam calcados em uma perspectiva médica com modelo de atendimento clínico (de 30-40 minutos uma vez por semana), que tende a minimizar a participação da família.

A literatura sugere que as práticas interventivas para populações com autismo são mais efetivas se forem de caráter intensivo (entre 25-40 horas semanais) e implementadas em situações naturais durante o período da primeira infância. Nesse contexto, a creche mostra-se um local adequado para o desenvolvimento de programas de intervenção precoce, pois além de acomodar crianças na mais tenra idade, oferta atendimento intensivo com período de duração de 4 - 7 horas diárias. Conforme discutido anteriormente, mudanças políticas e educacionais expressivas são, no entanto, demandadas para que essa prática se concretize na Educação Infantil brasileira.

\section{Referências}

Almeida, I. C. (2004). Intervenção precoce: Focada na criança ou centrada na família e na comunidade. Revista Análise Psicológica, 22 (1), 65-72.

Alves , R. C. P., Veríssimo, M. de La Ó. (2007). Os educadores de creche e o conflito entre cuidar e educar. Revista Brasileira de Crescimento e Desenvolvimento Humano, 17 (1), 13-25.

American Psychiatric Association APA (2013). DSM-V, Diagnostic and statistical manual of mental disorders (5th ed.). Washington, DC.

Andrade, L. B. P. (2010). Educação infantil: discurso, legislação e práticas institucionais São Paulo: Editora UNESP.

Bolsanello, M. A. (2003). Concepções sobre os procedimentos de intervenção e avaliação de profissionais em estimulação precoce. Educar em Revista, 22, 343-355.

Brasil (1998). Referencial curricular nacional para a educação infantil. V. 1, 2, e 3. Ministério da Educação. Brasília.

Brasil (2000). Referencial curricular nacional para a educaşão infantil: estratégias e Orientações para a educação de crianças com necessidades educacionais especiais. Ministério da Educação. Brasília. 
Brasil (2006). Política Nacional de Educação Infantil: pelo direito das crianças de zero a seis anos à educação. Ministério da Educação. Brasília.

Brasil (2013). Diretrizes de Atenção à Reabilitação da Pessoa com Transtornos do Espectro do Autismo. Secretaria de Atenção à Saúde, Série F. Comunicação e Educação em Saúde. 74, p. : il. Brasília

Briker, D. (2001). The natural environment: A useful construct? Infants and Young Children, 13 (4), 21 31. http:/ / dx.doi.org/10.1097/00001163-200113040-00008

Bronfenbrenner, U. (Ed.). (2005). Making buman beings buman: Bioecological perspectives on human development. Sage Publications. California.

Bueno, J. G., Meletti, S.M.F. (2011) Educação Infantil e Educação Especial: uma análise dos indicadores educacionais brasileiros. Revista Contraponto - Eletrônica, 11(3), 278-287.

Byington, T., Whitby. P. (2011). Empowering Families During the Early Intervention Planning Process. Young Exceptional Children, 14, 44-56. http:/ /dx.doi.org/10.1177/1096250611428878

Cunha, A. B. C., Benevides, J. (2012). Prática do psicólogo em intervenção precoce na saúde materno-infantil. Psicologia em Estudo, 17, 111-119. Maringá. http:/ /dx.doi.org/10.1590/S1413-73722012000100013

Dall' Aqua, M. F., Takiuchi, N., Zorzi, J. L. (2008). Efetividade de um treinamento de professores de uma escola de educação especial usando os princípios dos métodos Hanen e V.O.E.: veja, ouça e espere. Revista CEFAC, 10 (4). 433-442

Davies, A. J.; Kochhar, A. K. (2002). Manufacturing best practice and performance studies: a critique. International Journal of Operations \& Production Management, 22 (3), 289-305. http:/ /dx.doi.org/10.1108/01443570210417597

Dawson G, Osterling J. (1998). Early intervention in autism. In: M. Guralnick (Ed). The Effectiveness of Early Intervention (pp. 307-326): Baltimore: Brookes.

Dawson G, Rogers S, Munson J, et al (2010). Randomized controlled trial of the Early Start Denver Model: A developmental behavioral intervention for toddlers with autism: Effects on IQ, adaptive behavior, and autism diagnosis. Journal Pediatrics.125(1), 17-23. http:/ /dx.doi.org/10.1542/peds.2009-0958

Dunst, C., \& Bruder, M. B. (2002). Valued outcomes of service coordination, early intervention and natural environments. Exceptional Children, 68 (3), 361-375. http:/ / dx.doi.org/10.1177/001440290206800305

European Agency for Development in Special Needs Education (2010). Intervenção Precoce na Infância: Progressos e desenvolvimentos 2005-2010. Disponível em www.european-agency.org, acesso em 11.04.2013

Fombonne E. (2009) Epidemiology of pervasive developmental disorders. Pediatric Research Volume 65: 591-598. http://dx.doi.org/10.1203/PDR.0b013e31819e7203

Gable A. (2006). Influencing the Life Trajectories of Children with Asperger's Syndrome. International Journal of Disability, Development and Education, 53(1) 129-134. http:/ / dx.doi.org/10.1080/10349120500510131

Girolametto, L., Sussman, F., \& Weitzman, E. (2006). Using case study methods to investigate the effects of interactive interventions for children with autism spectrum disorders. Journal of Communication Disorders, 40, 470-492. http:/ /dx.doi.org/10.1016/j.jcomdis.2006.11.001

Greenspan, S. I., Wieder, S. (1998). The child with special needs: Intelectual and emotional growth. Reading, MA: Addison Wesley Longman.

Guralnick, M. J. (1998) Effectiveness of early intervention for vulnerable children: A developmental perspective. American Journal on Mental Retardation, 102, 319-345.

http://dx.doi.org/10.1352/0895-8017(1998)102<0319:EOEIFV>2.0.CO;2 
Johnson C. P., Myers S. M. (2007). American Academy of Pediatrics, Council on Children With Disabilities. Identification and evaluation of children with autism spectrum disorders. Journal of Pediatrics. 120 (5), 1183-1215.

Lampreia, C. (2007). A perspectiva desenvolvimentista para a intervenção precoce no autismo. Estudos de Psicologia, 24 (1), 105-114. http://dx.doi.org/10.1590/S0103-166X2007000100012

Love, J., Tarullo, L., Raikes, H. \& Chazan-Cohen R. (2008) Head Start: What do we need to know? In K. McCartney, \& D. Phillips (eds.) Blackwell handbook of early childhood development (pp. 550584). Malden: Blackwell Publishing.

Mahony G; Perales F. (2003) Using relationship-focused intervention to enhance the socialemotional functioning of young children with autism spectrum disorders. Topics Early Child Spec Educ 2003, 23, 77-89.

Mariotto, R. M. M. (2003). Atender cuidar e prevenir: A creche, a educação e a psicanálise. Estilos da Clinica, 8 (15), 34-47.

Matson, J. L., Konst, M. J. (2013). What is the evidence for long term effects of early autism interventions? Research in Autism Spectrum Disorders, (7), 475-479. http:/ / dx.doi.org/10.1016/j.rasd.2012.11.005

Mc Conachie, H., Randle, V., Hammal, D., Le Couteur, A. (2005). A controlled trial of a training course for parents of children with suspected autism spectrum disorder. Journal of Pediatrics, 147, 335-340. http://dx.doi.org/10.1016/j.jpeds.2005.03.056

Mendes, E. G. (2010). Inclusão marco zero: começando pelas creches. Araraquara, SP: Junqueira \& Marin.

National Research Council (2001). Educating Children with Autism. Committee on Educational Interventions for Children with Autism. Catherine Lord and James P. McGee, eds. Division of Behavioral and Social Sciences and Education. Washington.

Nunes, Debora R. P.; Azevedo, M. Q. O. ; Schmidt, C. (2013). Inclusão Educacional de pessoas com autismo no Brasil: uma revisão da literatura. Revista Educação Especial, 26, 557-572. http:/ /dx.doi.org/10.5902/1984686X10178

Nunes, L. R. (1995). Educação precoce para bebês de risco. In: B. Range (Org.). Psicoterapia comportamental e cognitiva (pp. 121-132). Campinas: Psy.

Nunes, L. R. (1996). Atendimento à criança carente e à criança de risco: análise do relato verbal de coordenadores e recreadores de creche. Em L .R. Nunes (Org.) Prevenção e intervenção em Educação Especial (p.56-79.). Rio de Janeiro: Associação Nacional de Pesquisa e PósGraduação em Psicologia.

Oliveira, Z. M. R. (1988). As creches no Brasil: mapeamento de uma trajetória. Revista Faculdade de Educação, 14 (1), p.43-52.

Pacheco, A. L. P. de B., Dupret, L. (2004). Creche: desenvolvimento ou sobrevivência? Psicologia USP, 15(3), 103-116. http://dx.doi.org/10.1590/S0103-65642004000200006

Pajareya, K., \& Nopmaneejumruslers, K. (2011). A pilot randomized controlled trial of DIR/Floortime ${ }^{\mathrm{TM}}$ parent training intervention for pre-school children with autistic spectrum disorders. Autism, 15 (5), 563-577. http://dx.doi.org/10.1177/1362361310386502

Paula, C. S, R., Sabrina H.; Fombonne, E., Mercadante, M. T. (2011). Brief Report: Prevalence of Pervasive Developmental Disorder in Brazil: A Pilot study. Journal of Autism and Developmental Disorders, 41, 1738-1742. http://dx.doi.org/10.1007/s10803-011-1200-6

Pereira, L.C.; Grave, M. (2012). Encaminhamento de crianças com necessidades educacionais especiais em idade de estimulação precoce a escolas de Educação Infantil de um município de médio porte do Vale dos Sinos. Revista Educação Especial 25 (42), 101-114.

Pereira, P. Matsukura, T. (2013). Inclusão escolar e educação infantil: um estudo de caso. Revista Educação Especial. 26 (45), 125-144. 
Prizant, B., Meyer, E. (1993). Socioemotional aspects of language and social-communication disorders in young children. American Journal of Speech-Language Pathology, 2, 56-71. http:/ / dx.doi.org/10.1044/1058-0360.0203.56

Rogers, S. J., Estes, A., Lord C., Vismara, L. A., Winter, J., Fitzpatrick, A., Guo, M., \& Dawson, G. (2012). Effects of a Brief Early Start Denver Model (ESDM)-Based Parent Intervention on Toddlers at Risk for Autism Spectrum Disorders: A Randomized Controlled Trial. Journal of the American Academy of Child \& Adolescent Psychiatry. 51 (10), 1052-1065. http://dx.doi.org/10.1016/j.jaac.2012.08.003

Sandall, S., Hemmeter, M., Smith, B., \& McLean, M. (2005). DEC recommended practices: A comprehensive guide. Denver: Division of Early Childhood for Exceptional Children.

Sandall, S., McLean, M., \& Smith, B. (2000). DEC recommended practices in early intervention: Early childhood special education. Denver: Division of Early Childhood for Exceptional Children.

Schmidt, C. (Org) (2013). Autismo, educação e transdisciplinaridade. São Paulo: Editora Papirus.

Schwartzman, J. S. \& Araújo, C. A. (Org.) (2011). Transtornos do Espectro do Autismo. São Paulo: Memnon Edições Científicas.

Simpson, R., et al. (2005). Autism Spectrum Disorders: Interventions and treatments for children and youth. Thousand Oaks, CA: Corwin Press.

Soejima, C. S.; Bolsanello, M. A. (2012). Programa de intervenção e atenção precoce com bebês na educação infantil. Educar em Revista. 43, 65-79. http:/ / dx.doi.org/10.1590/S010440602012000100006

Spaulding, L. S. (2009). Best practices and interventions in special education: How do we know what works? Teaching Exceptional Children Plus, 5(3), 1-13.

Strain, P. S., \& Hoyson, M. (2000). On the need for longitudinal, intensive social skill intervention: LEAP follow-up outcomes for children with autism as a case-in-point.Topics in Early Childhood Special Education, 20,116-122. http:/ / dx.doi.org/10.1177/027112140002000207

Strain, P. S.; Bovey II, E. H. (2011). Randomized, controlled trial of the leap model of early intervention for young children with autism spectrum disorders. Topics in Early Childhood Special Education, 31 (3), 133-154. http://dx.doi.org/10.1177/0271121411408740

Sussman, F. (2004). More Than Words. Hanen Centre, Toronto, Canadá.

Thompson, T. (2011). Individualized autism intervention for young children: Blending discrete trial and naturalistic strategies. Baltimore, MD: Paul H. Brookes.

Vismara L.A.; Colombi C.; Rogers S.J. (2009) Can 1 hour per week of therapy lead to lasting changes in young children with autism? Autism. 13(1), 93-115. http://dx.doi.org/10.1177/1362361307098516

Williams, L. C. A.; Aiello, A. L. R. (2001). O inventário portage operacionalizado: intervenção com famílias. São Paulo: Memnon. 


\section{Sobre as Autoras}

\section{Débora Regina de Paula Nunes}

Programa de Pós-Graduação em Educação da Universidade Federal do Rio Grande do Norte (PPGED/UFRN). deboranunes@ufrnet.br

Ph.D. em Educação Especial pela Florida State University, com mestrado em Educação pela Universidade do Estado do Rio de Janeiro (UERJ) e graduação em Psicologia pela Universidade

Federal do Rio de Janeiro (UFRJ). É docente dos Programas de Graduação e Pós-Graduação em Educação da Universidade Federal do Rio Grande do Norte. Atualmente é editora adjunta da Revista Brasileira de Educação Especial e integrante da Base de Pesquisa sobre Educação de Pessoas com Necessidades Especiais (UFRN). Desenvolve pesquisas sobre autismo, comunicação alternativa e adaptação curricular.

\section{Eliana Rodrigues Araújo.}

Comissão Permanente de Apoio a Estudantes com Necessidades Educacionais Especiais da Universidade Federal do Rio Grande do Norte (CAENE/UFRN).

eliana_natal@yahoo.com.br

Mestre em Educação pela Universidade Federal do Rio Grande do Norte (UFRN), com mais de 15 anos de experiência em intervenção precoce de crianças com autismo no contexto domiciliar. Atualmente é pedagoga na CAENE/UFRN, onde desenvolve estudos sobre a inclusão de alunos com autismo e atua como mediadora no processo de inclusão dos estudantes com autismo e altas habilidades na Universidade.

\section{Sobre as Editoras Convidadas}

\section{Márcia Denise Pletsch}

Professora do Programa de Pós-Graduação em Educação, Contextos Contemporâneos e Demandas Populares (PPGEduc) na linha de pesquisa Estudos Contemporâneos e Práticas Educativas e do Departamento Educação e Sociedade da Universidade Federal Rural do Rio de janeiro. marciadenisepletsch@gmail.com

É pesquisadora na área de Educação Especial, atuando na formação de professores e de novos pesquisadores. É líder do Grupo de Pesquisa (CNPq) Observatório de Educação Especial e inclusão escolar: práticas curriculares e processos de ensino e aprendizagem e, por meio de convênio interinstitucional entre a UFRRJ e a Universidade do Estado do Rio de Janeiro (UERJ), também é líder do grupo de pesquisa Inclusão e aprendizagem de alunos com necessidades educacionais especiais: práticas pedagógicas, cultura escolar e aspectos psicossociais. Atualmente, coordena o Programa Observatório da Educação da CAPES com projeto de pesquisa em rede na área de deficiência intelectual envolvendo a Universidade Estadual de Santa Catarina (UDESC), Universidade do Vale do Itajaí (UNIVALI); e coordena também pesquisas financiadas pela FAPERJ na área de deficiência múltipla. É autora do livro "Repensando a inclusão escolar: diretrizes políticas, práticas curriculares e deficiência intelectual" e, em colaboração com Rosana Glat, do livro "Inclusão escolar de alunos com necessidades especiais". Organizou em parceria com outros pesquisadores, entre outros, os livros "Estratégias educacionais diferenciadas para alunos com necessidades especiais" e "Educação Especial e inclusão escolar: 
reflexões sobre o fazer pedagógico". Tem mais de vinte artigos publicados em revistas científicas nacionais e internacionais.

Geovana Mendonça Lunardi Mendes - Professora do Programa de Pós-Graduação em Educação e do Departamento de Pedagogia da Universidade do Estado de Santa Catarina (PGEUDESC). geolunardi@gmail.com

E pesquisadora na área de Educação Especial e dos Estudos Curriculares. Realizou Pós-Doutorado na Argentina e nos Estados Unidos da América, na área de Currículo e Novas Tecnologias, na Universidad de San Andres em Buenos Aires e em Ashland University, em Ohio. É pesquisadora coordenadora de diferentes projetos de investigação e participa como pesquisadora convidada em projetos de pesquisa nacionais e internacionais. Suas pesquisas e produções têm sido voltadas para área de Currículo e práticas escolares, em especial, as questões relativas as mudanças, novas tecnologias e inovações curriculares no espaço escolar, e também as práticas curriculares voltadas a inclusão de sujeitos com deficiência. Atualmente é a Coordenadora Nacional do Consórcio "Educação e Diversidade" do programa CAPES. FIPSE de Cooperação Internacional, envolvendo a Universidade Federal Rural do Rio de Janeiro, no Brasil e Georgetown College, Ashland University e Brighman Young University nos Estados Unidos e também do Projeto de Pesquisa: Aulas conectadas: mudanças curriculares e aprendizagem colaborativa nas escolas do PROUCA em Santa Catarina, com financiamento do CNPq e do Projeto Observatório de Práticas Escolares com financiamento da FAPESC. É coordenadora do Programa de Pós-graduação em Educação, Mestrado e Doutorado, da FAED, UDESC. Coordena o Observatório da Educação: Tablets, Computadores e Laptops, aprovado no Edital OBEDUC/CAPES. Entre suas atuais produções podem ser destacadas o livro intitulado "Objetos Pedagógicos: uma experiência inclusive em oficinas de Artes", em parceria com mais duas autoras e a organização do Livro "Deficiência e Escolarização: novas perspectivas de analise", hoje na segunda edição. É autora de inúmeros capítulos e artigos publicados em periódicos.

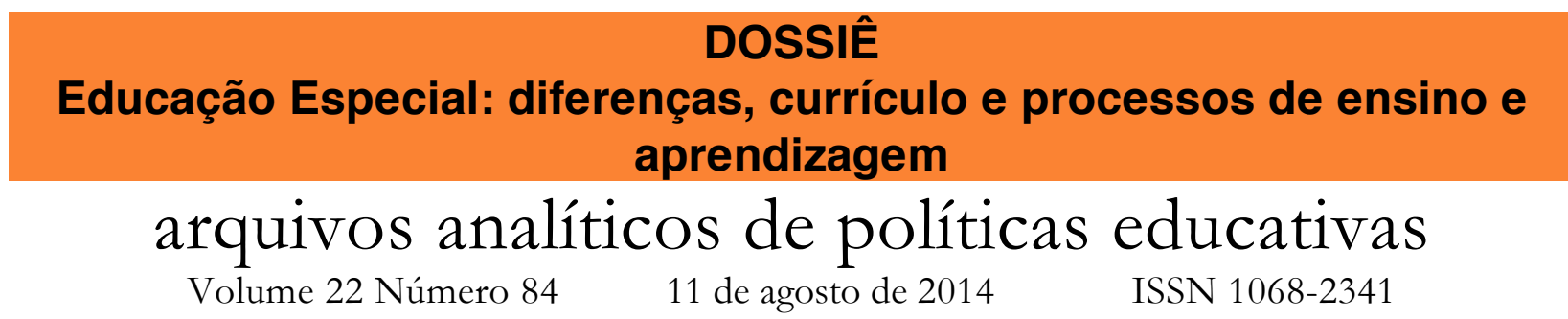

\footnotetext{
(c)

SORERIGHIS RESERVED O Copyright e retido pelo/a o autor/a (ou primeiro co-autor) que outorga o direito da primeira publicação à revista Arquivos Analíticos de Políticas Educativas. Más informação da licença de Creative Commons encontram-se em http://creativecommons.org/licenses/by-nc-nd/2.5. Qualquer outro uso deve ser aprovado em conjunto pelo/s autor/es e por AAPE/EPAA. AAPE/EPAA é publicada por Mary Lou Fulton
} Institute Teachers College da Arizona State University. Os textos publicados em AAPE são indexados por 
CIRC (Clasificación Integrada de Revistas Científicas, Espanha) DIALNET (Espanha),Directory of Open Access Journals, Education Full Text (H.W. Wilson), EBSCO Education Research Complete, , ERIC, , QUALIS A2 (Brasil), SCImago Journal Rank; SCOPUS, SOCOLAR (China). Contribua com comentários e sugestões a http://epaa.info/wordpress/ ou para Gustavo E. Fischman fischman@asu.edu.

Curta a nossa comunidade EPAA's Facebook https://www.facebook.com/EPAAAAPE e Twitter feed@epaa_aape. 


\section{arquivos analíticos de políticas educativas conselho editorial}

Editor: Gustavo E. Fischman (Arizona State University) Editores Associados: Rosa Maria Bueno Fisher e Luis A. Gandin

(Universidade Federal do Rio Grande do Sul)

Dalila Andrade de Oliveira Universidade Federal de Minas Gerais, Brasil

Paulo Carrano Universidade Federal Fluminense, Brasil

Alicia Maria Catalano de Bonamino Pontificia Universidade Católica-Rio, Brasil

Fabiana de Amorim Marcello Universidade Luterana do Brasil, Canoas, Brasil

Alexandre Fernandez Vaz Universidade Federal de Santa Catarina, Brasil

Gaudêncio Frigotto Universidade do Estado do Rio de Janeiro, Brasil

Alfredo M Gomes Universidade Federal de Pernambuco, Brasil

Petronilha Beatriz Gonçalves e Silva Universidade Federal de São Carlos, Brasil

Nadja Herman Pontificia Universidade Católica -Rio Grande do Sul, Brasil

José Machado Pais Instituto de Ciências Sociais da Universidade de Lisboa, Portugal

Wenceslao Machado de Oliveira Jr. Universidade Estadual de Campinas, Brasil
Jefferson Mainardes Universidade Estadual de Ponta Grossa, Brasil

Luciano Mendes de Faria Filho Universidade Federal de Minas Gerais, Brasil

Lia Raquel Moreira Oliveira Universidade do Minho, Portugal

Belmira Oliveira Bueno Universidade de São Paulo, Brasil

António Teodoro Universidade Lusófona, Portugal

Pia L. Wong California State University Sacramento, U.S.A

Sandra Regina Sales Universidade Federal Rural do Rio de Janeiro, Brasil

Elba Siqueira Sá Barreto Fundação Carlos Chagas, Brasil

Manuela Terrasêca Universidade do Porto, Portugal

Robert Verhine Universidade Federal da Bahia, Brasil

Antônio A. S. Zuin Universidade Federal de São Carlos, Brasil 


\section{education policy analysis archives editorial board}

Editor Gustavo E. Fischman (Arizona State University)

Associate Editors: Audrey Amrein-Beardsley (Arizona State University), Rick Mintrop, (University of California, Jeanne M. Powers (Arizona State University)

Jessica Allen University of Colorado, Boulder

Gary Anderson New York University

Michael W. Apple University of Wisconsin, Madison

Angela Arzubiaga Arizona State University

David C. Berliner Arizona State University

Robert Bickel Marshall University

Henry Braun Boston College

Eric Camburn University of Wisconsin, Madison

Wendy C. Chi* University of Colorado, Boulder

Casey Cobb University of Connecticut

Arnold Danzig Arizona State University

Antonia Darder University of Illinois, UrbanaChampaign

Linda Darling-Hammond Stanford University

Chad d'Entremont Strategies for Children

John Diamond Harvard University

Tara Donahue Learning Point Associates

Sherman Dorn University of South Florida

Christopher Joseph Frey Bowling Green State University

Melissa Lynn Freeman* Adams State College

Amy Garrett Dikkers University of Minnesota

Gene V Glass Arizona State University

Ronald Glass University of California, Santa Cruz

Harvey Goldstein Bristol University

Jacob P. K. Gross Indiana University

Eric M. Haas WestEd

Kimberly Joy Howard* University of Southern California

Aimee Howley Ohio University

Craig Howley Ohio University

Steve Klees University of Maryland

Jaekyung Lee SUNY Buffalo
Christopher Lubienski University of Illinois, UrbanaChampaign

Sarah Lubienski University of Illinois, UrbanaChampaign

Samuel R. Lucas University of California, Berkeley

Maria Martinez-Coslo University of Texas, Arlington

William Mathis University of Colorado, Boulder

Tristan McCowan Institute of Education, London

Heinrich Mintrop University of California, Berkeley

Michele S. Moses University of Colorado, Boulder

Julianne Moss University of Melbourne

Sharon Nichols University of Texas, San Antonio

Noga O'Connor University of Iowa

João Paraskveva University of Massachusetts, Dartmouth

Laurence Parker University of Illinois, UrbanaChampaign

Susan L. Robertson Bristol University

John Rogers University of California, Los Angeles

A. G. Rud Purdue University

Felicia C. Sanders The Pennsylvania State University

Janelle Scott University of California, Berkeley

Kimberly Scott Arizona State University

Dorothy Shipps Baruch College/CUNY

Maria Teresa Tatto Michigan State University

Larisa Warhol University of Connecticut

Cally Waite Social Science Research Council

John Weathers University of Colorado, Colorado Springs

Kevin Welner University of Colorado, Boulder

Ed Wiley University of Colorado, Boulder

Terrence G. Wiley Arizona State University

John Willinsky Stanford University

Kyo Yamashiro University of California, Los Angeles

* Members of the New Scholars Board 


\section{archivos analíticos de políticas educativas consejo editorial}

Editor: Gustavo E. Fischman (Arizona State University)

Editores. Asociados Jason Beech (Universidad de San Andrés) Alejandro Canales (UNAM) y Jesús Romero Morante (Universidad de Cantabria)

Armando Alcántara Santuario Instituto de Investigaciones sobre la Universidad y la Educación, UNAM México

Claudio Almonacid Universidad Metropolitana de Ciencias de la Educación, Chile

Pilar Arnaiz Sánchez Universidad de Murcia, España

Xavier Besalú Costa Universitat de Girona, España Jose Joaquin Brunner Universidad Diego Portales, Chile

Damián Canales Sánchez Instituto Nacional para la Evaluación de la Educación, México

María Caridad García Universidad Católica del Norte, Chile

Raimundo Cuesta Fernández IES Fray Luis de León, España

Marco Antonio Delgado Fuentes Universidad Iberoamericana, México

Inés Dussel FLACSO, Argentina

Rafael Feito Alonso Universidad Complutense de Madrid, España

Pedro Flores Crespo Universidad Iberoamericana, México

Verónica García Martínez Universidad Juárez Autónoma de Tabasco, México

Francisco F. García Pérez Universidad de Sevilla, España

Edna Luna Serrano Universidad Autónoma de Baja California, México

Alma Maldonado Departamento de Investigaciones Educativas, Centro de Investigación y de Estudios Avanzados, México

Alejandro Márquez Jiménez Instituto de Investigaciones sobre la Universidad y la Educación, UNAM México

José Felipe Martínez Fernández University of California Los Angeles, USA
Fanni Muñoz Pontificia Universidad Católica de Perú

Imanol Ordorika Instituto de Investigaciones Economicas - UNAM, México

Maria Cristina Parra Sandoval Universidad de Zulia, Venezuela

Miguel A. Pereyra Universidad de Granada, España

Monica Pini Universidad Nacional de San Martín, Argentina

Paula Razquin UNESCO, Francia

Ignacio Rivas Flores Universidad de Málaga, España

Daniel Schugurensky Arizona State University

Orlando Pulido Chaves Universidad Pedagógica Nacional, Colombia

José Gregorio Rodríguez Universidad Nacional de Colombia

Miriam Rodríguez Vargas Universidad Autónoma de Tamaulipas, México

Mario Rueda Beltrán Instituto de Investigaciones sobre la Universidad y la Educación, UNAM México

José Luis San Fabián Maroto Universidad de Oviedo, España

Yengny Marisol Silva Laya Universidad Iberoamericana, México

Aida Terrón Bañuelos Universidad de Oviedo, España

Jurjo Torres Santomé Universidad de la Coruña, España

Antoni Verger Planells University of Amsterdam, Holanda

Mario Yapu Universidad Para la Investigación Estratégica, Bolivia 